\title{
Desigualdades regionais e o papel dos recursos federais no SUS: fatores políticos condicionam a alocação de recursos?
}

\author{
Regional inequalities and the role of federal resources in the SUS: \\ do political factors affect the allocation of funds?
}

Gregory dos Passos Carvalho (http://orcid.org/0000-0003-3173-9732) ${ }^{1}$

${ }^{1}$ Instituto de Ciência Política, Universidade de Brasília. Prédio IPOL/IREL - IPOL, UNB Área 1.70904970. Brasília DF Brasil. gcarvalho.pol@gmail.com

\begin{abstract}
The research problem in this paper is a discussion of the political factors affecting the allocation of federal resources in the SUS (Unified Health System) and its effects on regional inequalities in Brazil. The main objective was to explore the influence of political factors as criteria for the distribution of federal funds, considering the regional inequalities of the country. A mixed method was used by collecting quantitative data to study the allocation of resources for each unit of the Federation, from 2003 to 2017, in search of outliers, which require more in-depth explanation based on qualitative research methods. The fuzzyset QCA software was applied in the qualitative research phase for identification of technical and political conditions, where required and/or sufficient, for an atypical distribution of resources from one year to another. The quantitative analysis demonstrated the decrease of regional inequalities in allocation of resources to States in the North and Northeast. However, when studying the constraints of the distribution of the funds for the States of these regions, qualitative analysis demonstrated the influence of political factors on the allocation of resources, rather than purely technical factors.
\end{abstract}

Key words Political factors, Resource allocation in health, Political science, Data analysis, Qualitative comparative analysis
Resumo O presente trabalho apresenta como problema de pesquisa a discussão de fatores políticos enquanto condicionantes na alocação de recursos federais no SUS e seus efeitos nas desigualdades regionais. Tem como objetivo principal explorar a interveniência de fatores políticos como critérios na distribuição de recursos federais, considerando as desigualdades regionais no país. Utilizou-se método misto, por meio de coleta de dados quantitativos para se estudar a alocação dos recursos para as unidades da federação entre 2003 e 2017, em busca de casos emblemáticos (outliers) que demandem explicações mais profundas com base em métodos qualitativos. Aplicou-se o método fuzzy-set QCA na etapa qualitativa de pesquisa para identificação de condições técnicas e políticas, enquanto necessárias e/ou suficientes, para uma distribuição atípica de recursos de um ano para o outro. Como resultado, a análise quantitativa demonstrou a diminuição das desigualdades regionais na alocação de recursos de média e alta complexidade para estados das regiões Norte e Nordeste. Porém, ao estudar as condicionantes da distribuição dos recursos para os estados dessas regiões, a análise qualitativa demonstrou a influência de fatores políticos, e não somente fatores técnicos.

Palavras-chave Fatores politicos, Recursos financeiros em saúde, Ciência política, Análise de dados, Análise qualitativa comparativa 


\section{Introdução}

O presente trabalho traz à tona o debate acerca da possibilidade de fatores políticos serem critérios para distribuição dos recursos federais a estados e municípios no Sistema Único de Saúde (SUS).

Recentemente, um estudo sobre transferências de recursos no SUS identificou alinhamento partidário entre prefeitos e o presidente da República como fator estatisticamente significante na alocação de recursos para municípios, indicando que a distribuição de recursos do SUS possa ser explicada por fatores políticos ${ }^{1}$. Porém, para a literatura especializada em transferências intergovernamentais, os critérios de partilha dos recursos do SUS estruturados a partir dos anos 1990 colocariam a saúde em situação distinta do que ocorre nas transferências voluntárias da união (TVUs) - essas sim possuindo um considerável componente político na sua alocação ${ }^{2,3}$, em que emergem a influência de fatores vinculados ao presidencialismo de coalizão e às eleições.

Em decorrência dessa distinção, praticamente não há discussão acerca de fatores políticos influenciarem na alocação de recursos do SUS. Porém, deve-se destacar que a literatura no campo da saúde indica que os critérios de distribuição de recursos previstos nos marcos legais do SUS, que contribuiriam para diminuição das desigualdades regionais e maior transparência, ainda não foram efetivamente implementados, seja por motivações operacionais ou mesmo por falta de vontade política ${ }^{4}$.

Diante dessas divergências e tomando por base os achados de efeitos políticos na alocação de recursos do SUS ${ }^{1}$ e a ausência de aplicação dos critérios dispostos nos marcos legais ${ }^{4}$, pode-se reconsiderar a distinção entre as TVUs e as transferências do SUS, tema esse ainda incipiente e que pode ser mais bem estudado.

Dito isso, este trabalho apresenta como problema de pesquisa a discussão de fatores políticos enquanto condicionantes na alocação de recursos federais no SUS e seus efeitos nas desigualdades regionais. Assim, tem-se como objetivo principal explorar a interveniência de fatores políticos como critérios na distribuição de recursos federais no SUS, considerando as desigualdades regionais no país. Em síntese, será realizada a aplicação de método misto, tendo como objeto de análise a alocação dos recursos do bloco da média e alta complexidade em saúde, dada a representatividade desse grupo de ações e serviços no total dos recursos do SUS transferidos para as unidadesda federação ${ }^{4}$. Por meio de técnicas de estatística descritiva, será avaliado o padrão da distribuição de recursos ao longo de 15 anos (2003 a 2017), "clusterizando"as UFs conforme elas recebam mais ou menos recursos federais, além de avaliar avariação percentual de um ano para o outro para a identificação de eventuais outliers. Observando-se a identificação de outliers, esses serão mais bem estudados por meio de análise comparada qualitativa, especificamente com o método fuzzy-setQCA, que possibilita a identificação de condições necessárias e/ ou suficientes para explicação de um fenômeno em estudo. Em suma, os achados a partir dos métodos quantitativos apontarão os casos que demandarão análises mais profundas com base em métodos qualitativos.

A segunda e próxima seção apresentarão a discussão teórica em torno do tema transferências intergovernamentais no Brasil, considerando a literatura da ciência política e também as discussões específicas da saúde. A terceira seção apresentará as considerações metodológicas gerais. Uma quarta seção realizará a análise quantitativa e discutirá os seus resultados. A quinta seção, a partir dos dados extraídos da primeira análise, aplicará o método qualitativo e então discutirá os seus achados. Por fim, uma sexta seção realizará um balanço geral dos resultados identificados.

\section{Discussões sobre transferências intergovernamentais no Brasil e na saúde}

Quando se estuda as TVUs, grupo em que as transferências legais do SUS não se enquadram, artigos que partem de referenciais teóricos da ciência política discutem quais fatores políticos estão associados à alocação de recursos federais para os demais entes da federação ${ }^{2,3,5,6}$. Identificam-se a distribuição de recursos federais prevalente em anos eleitorais, indicando-o como recurso de estratégia eleitoral, e também a relação entre número de parlamentares aliados do governo federal na bancada estadual e uma maior chance de se obter recursos ${ }^{2}$. Identificase também como significante o alinhamento de prefeitos com o partido do presidente ${ }^{3}$. Outro fator político identificado é a relação com eleições municipais ${ }^{5}$.

Essas análises possuem um olhar centrado nas TVUs. Outra publicação, ao estudar valores atípicos no total de todos os recursos correntes por habitante transferidos entre 1985 e 2004 (e não somente TVUs), também conclui que critérios políticos se sobrepõem a critérios de equida- 
de, observando-se o efeito prevalente de bancada estadual aliada do governo federal frente a variáveis de redução das desigualdades regionais ${ }^{6}$.

Por fim, um outro estudo, que observa especificamente municípios e as transferências do SUS, identifica como significante o alinhamento partidário entre prefeito e presidente da República, além de considerável relação entre receita municipal e maior volume de recursos federais transferidos, apontando um efeito não desejável no que se refere à redução de desigualdades regionais ${ }^{1}$.

Nesse ponto, observando-se especificamente a saúde, há um conjunto de temas a ser considerado. Inicialmente, cabe destacar a discussão de redução das desigualdades regionais que se relaciona a aspectos das relações federativas e da coordenação de políticas públicas em contextos de descentralização política. Em teoria, nos processos de descentralização política a coordenação federativa surge como relevante na expectativa de se garantir a redução de inequidades e desigualdades regionais, destacando-se o uso de instrumentos como as transferências intergovernamentais ${ }^{7}$. A princípio, a redistribuição de recursos financeiros federais poderia servir ao propósito de equalizar desequilíbrios da federação brasileira, sejam eles verticais ou horizontais ${ }^{8}$.

Para exemplificar, teria ocorrido um conjunto de avanços na redução das desigualdades regionais no SUS no início dos anos 2000, em que a distribuição mais equitativa de recursos estava no centro das estratégias de financiamento utilizadas pelo Ministério da Saúde ${ }^{9}$. Os avanços na redistribuição de recursos entre estados, observando valores per capita, teriam ocorrido tanto no piso de atenção básica (PAB) quanto nas ações e serviços de média e alta complexidade (MAC) - essa última corresponde a quase 70\% do total dos recursos federais transferidos ${ }^{4,10}$. De fato, ao observar os dados apresentados, constata-se uma considerável variação positiva na alocação de recursos de MAC para os estados antes menos favorecidos, ainda que estados das regiões Norte e Nordeste figurem com os menores per capitas 9.

Entretanto, é preciso destacar que não há consenso sobre a redução das desigualdades, e uma publicação sistematiza amplamente a divergência de entendimentos acadêmicos relacionada à alocação de recursos no SUS ${ }^{8}$. Como exemplo, a depender do período e dos recursos estudados, tem publicação que indica maior necessidade de se estabelecer uma alocação mais equitativa ${ }^{11}$. Por outro lado, quando se observa recursos por região geográfica comparativamente à população residente, não haveria grandes distorções ${ }^{12}$, e em outros casos haveria uma queda na desigualdade de distribuição de recursos entre municípios, inclusive com queda do coeficiente de Gini, especificamente nos recursos do $\mathrm{PAB}^{13}$.

Em complemento, deve-se se questionar a real capacidade de o Ministério da Saúde diminuir as desigualdades regionais, especialmente em um contexto em que as ações de MAC são dependentes da existência de prestadores privados, esses em geral concentrados em municípios com renda mais elevada ${ }^{14}$. Nesse contexto, a alocação de recursos de MAC está relacionada à série histórica do gasto em serviços ambulatoriais e hospitalares e, mesmo com os mecanismos regulares fundo a fundo, reproduz as condições do passado, privilegiando a capacidade de oferta de serviços e não atacando as desigualdades, inclusive não havendo espaço para regulamentação do artigo 17 da Lei Complementar no 141/2012, que estabelece critérios mais equitativos de alocação de recursos, com foco nas necessidades de saúde ${ }^{15}$.

Destaca-se que a alocação de recursos conforme a legislação federal não vem sendo cumprida desde os anos 1990 quando se observa a Lei $n^{\circ} 8.080 / 1990$, que já teria apontado critérios para alocação equitativa, e a Lei no 8.142/1990, que prevê a distribuição per capita ${ }^{4}$. Haveria na prática um conjunto de dificuldades para se operacionalizar as bases legais do SUS, como questões operacionais, falta de priorização ou mesmo vontade política ${ }^{12}$. Outro impeditivo seria a ausência de recursos adicionais para que uma eventual redistribuição possa ser realizada sem perda financeira para nenhum ente federado ${ }^{12}$. Haveria também limitações considerando-se o contexto de disparidades socioeconômicas relevantes no país, que impactaria a possibilidade de prestação dos serviços de saúde em determinadas localida$\operatorname{des}^{16}$.

Nesse cenário, diversos critérios, definidos por portarias do Ministério da Saúde, são utilizados para as transferências do SUS, no vácuo do que é disposto em lei, e essa enormidade de regras tornaria inclusive o acompanhamento e a compreensão dos repasses uma tarefa comple$\mathrm{xa}^{17}$. Em consulta às normativas vigentes, quase 1.200 artigos estabelecem incentivos e condicionam os repasses de recursos às estratégias definidas nacionalmente ${ }^{18}$. O elevado arcabouço normativo levaria também a contradições, onde convivem incentivos explícitos que buscam uma maior eficiência alocativa e produtiva e a redução de desigualdades, mas também incentivos 
implícitos que contradizem esses objetivos, perpetuando desigualdades, especificamente na $\mathrm{MAC}^{14,19}$. É nesse contexto que, inesperadamente, nas palavras dos autores do artigo identificado, fatores políticos poderiam explicar a alocação de recursos no SUS - notadamente, o alinhamento partidário entre Presidência da República e prefeituras ${ }^{1}$. Nesse tema, é preciso considerar aspectos do modelo presidencialista multipartidário brasileiro, em que a necessidade de se construir apoio político é exercida considerando diversos instrumentos: alocação de recursos financeiros, estabelecimento de acordos sobre políticas públicas e, mais recentemente, discussões relacionadas à nomeação para cargos públicos com o objetivo de viabilizar direcionamento de recursos para redutos políticos de um partido ou de um político em específico por meio do controle de poucos cargos estratégicos ${ }^{20}$. Adiciona-se nessa discussão a noção de que muitas das decisões políticas são tomadas não pelo ministério temático, mas no chamado núcleo da Presidência da República ${ }^{20}$.

Feitas essas considerações, a discussão aqui proposta de estudar os fatores que condicionam a alocação de recursos do SUS demanda uma agenda robusta de pesquisa que possa compreender a presença de fatores políticos permeando essa distribuição.

\section{Aspectos metodológicos}

Quanto aos aspectos metodológicos, o presente trabalho optou por realizar uma combinação de métodos do tipo explicação sequencial ${ }^{21}$. A ideia central é, a partir de uma análise quantitativa inicial, identificar casos atípicos no recebimento de recursos federais. Feito isso, tentará identificar possíveis condições necessárias e suficientes para esse fenômeno, considerando técnicas qualitativas.

A opção de dedicar parte desse estudo a uma análise mais específica de outliers no recebimento de recursos emerge da necessidade de se reconsiderar a distinção entre as TVUs e as transferências do SUS, indicando-se uma análise exploratória acerca de eventuais condicionantes políticos na alocação de recursos do SUS. O uso de casos atípicos é adequado para a realização de análises exploratórias e construção de teorias, observando-se o que se chama de tipos ideais, que inclusive ajudam a refinar e testar modelos estatísticos futuros ${ }^{22}$. Não serão utilizadas técnicas quantitativas para análise de causas de situações atípicas por entender que essa não é uma escolha muito aconselhável na tradição quantitativa ${ }^{23}$, sendo mais recomendada as técnicas qualitativas ${ }^{22}$.

Dito isso, e considerando como problema de pesquisa a discussão de fatores políticos enquanto condicionantes na alocação de recursos federais no SUS e seus efeitos nas desigualdades regionais, as questões deste artigo são: quais fatores (técnicos e/ou políticos) são condições necessárias e/ou suficientes para a alocação atípica de recursos no SUS para uma ou mais UFs? Fatores políticos podem ser considerados como condições necessárias e/ou suficientes? Há interação entre diversos fatores, sejam eles políticos ou técnicos?

A identificação de casos atípicos será realizada por meio de análises quantitativas, considerando os dados do Fundo Nacional de Saúde (FNS), montando um banco com o total de repasses realizados para cada uma das 27 Unidades da Federação (UF) ao longo de 15 anos (2003 a 2017) e observando-se especificamente os repasses associados ao chamado bloco da média e alta complexidade, por se tratar do maior volume de recursos repassados aos estados e municípios (quase $70 \%$ ) e por não ter tido em sua história a implementação direta de critérios populacionais ou ponderados por condições socioeconômicas, como é o caso do PAB. Não serão considerados os repasses de MAC advindos de emendas parlamentares (que a partir de 2015 puderam complementar os recursos federais de MAC de estados e municípios).

Para melhor compreensão da alocação de recursos em MAC ao longo dos anos serão realizadas duas análises no software $\mathrm{R}^{24}$ : clusterização dos casos e identificação de casos outliers.

Em suma, para cada ano os valores de repasses serão corrigidos com o IPCA, considerandose o ano de 2017. A partir daí, será calculado o per capita da distribuição de recursos para cada uma das 27 unidades da federação, dividindo-se o valor total de repasses do MAC pela população IBGE de cada UF em cada ano em questão.

Em seguida será realizada uma análise com base no método k-means de clusterização dos casos, ano a ano, separando-os em três grupos: per capita mais baixo, per capita mais elevado e per capita intermediário. Isso ajuda a identificar eventuais UFs que possuem um per capita mais elevado ao longo dos 15 anos, outros que possuem per capita mais baixo e também aqueles que possuem oscilação ano a ano, contribuindo para observar as desigualdades no país ao longo do período estudado.

Posteriormente, será calculada a diferença em termos percentuais da variação do per capita do 
MAC de cada estado de um ano para o outro. Assim, por meio de técnicas de estatística descritiva, será avaliado o padrão da distribuição de casos (variação percentual do per capita do MAC de um ano para o outro) e identificação de eventuais outliers em cada ano separadamente, observando todas as UFs por meio de gráfico boxplot. Assim, ao final desse processo, tem-se a identificação de outliers, que seriam UFs que tiveram variação do per capita do MAC de um ano para o outro fora do padrão de cada ano.

Após esse exercício de natureza quantitativa, será realizada a análise qualitativa desses dados, observando-se os casos outliers para identificar fatores técnicos e/ou políticos que expliquem o aumento atípico no recebimento de recursos. Entre as possibilidades metodológicas de natureza qualitativa, entende-se que a técnica qualitative comparative analysis (QCA), mais especificamente o seu desdobramento fuzzy-set QCA, adequa-se bem aos propósitos e à versatilidade exigida neste estudo.

No debate metodológico da ciência política, pode-se atribuir aos métodos qualitativos uma orientação matemática implícita, que envolve a análise de necessidade e suficiência de condições para um determinado fenômeno em estudo, em que a técnica QCA retrata mais evidentemente esses aspectos matemáticos ${ }^{22}$. Trata-se de uma técnica versátil, desenvolvida inicialmente para aplicações com número de casos pequeno e médio (entre 15 e 50 casos) ${ }^{25}$. Com a evolução do método, o mesmo pode ser aplicado para um número grande de casos ${ }^{26}$. Trata-se, ainda, de uma técnica exploratória/interpretativa, questões essas que refletem a lógica e o espírito da pesquisa qualitativa ${ }^{26}$ e que se relaciona aos objetivos e perspectivas deste trabalho. Sua aplicação será desenvolvida também por meio do software $\mathrm{R}^{27}$.

Cabe esclarecer que, diferentemente das técnicas quantitativas, a questão-chave do QCA não é o quão forte é uma variável, mas sim como diferentes condições isoladamente ou combinadamente explicam o fenômeno em estudo, fazendo emergir aspectos de multiplicidade e complexidade causais $^{26}$. De modo complementar, o método permite qualificar cada caso em estudo e, considerando a combinação de condições, obtém-se um conhecimento mais aprofundado dos casos estudados ${ }^{26}-$ aspectos típicos das pesquisas qualitativas ${ }^{22}$.

Para viabilizar esse tipo de análise, o método QCA trabalha tanto com lógica binária, para valorar cada condição analisada, isto é, as condições assumem valores "0" ou "1" de maneira dicotômica, quanto com valores de associação entre " 0 " e "1", oferecendo uma vantagem de enquadramento dos casos às condições, conforme graus de pertencimento (mais próximo de "1" ou mais próximo de "0"), chamado de fuzzy-set QCA ${ }^{25.26}$.

Com isso, permite-se descrição e análise de relações lógicas embutidas em tabelas verdade, em que ferramentas set-theoretic (teoria de conjuntos) permitem avaliar relações entre condições e resultados sob a ótica de necessidade e suficiência ${ }^{22}$. Nesse tipo de análise pode surgir, por exemplo, uma condição que isoladamente não é suficiente para explicar um fenômeno mas que, numa combinação de condições, é parte necessária para que essa combinação seja suficiente - o que se chama de "INUS condition"22,26.

$\mathrm{Na}$ análise QCA as condições (variáveis explicativas) serão trabalhadas para cada UF e ano de análise, conforme o Quadro 1, que sistematiza a condição, o seu indicador, como ele será visualizado nos resultados da análise, a referência teórica e a sua operacionalização.

No que se refere ao resultado estudado (variável dependente), esse será trabalhado de maneira dicotômica, em que 1 corresponde aos casos que se enquadrem como outliers e 0 corresponde aos casos que não forem enquadrados como outliers.

Conforme a lógica de análise do método fuzzy-set QCA (teoria dos conjuntos), analisam-se: a) condições causais compartilhadas por casos com o mesmo resultado, ajudando a avaliar condições necessárias (aquelas que são requisitos ou indispensáveis para que determinado resultado aconteça); b) casos com as mesmas condições causais, observando em que nível eles compartilham o mesmo resultado, o que ajuda a avaliar condições suficientes (aquelas que asseguram ou geralmente levam a determinado resultado).

Existe um conjunto de recomendações para a realização das análises de necessidade e suficiência de condições que serão consideradas nesse trabalho ${ }^{29,30}$. Cabe registrar que uma condição, ou mesmo uma combinação de condições (preceito de complexidade causal), só será considerada suficiente quando a sua associação com o enquadramento outlier - consistência da relação - assumir valor maior ou igual a 0,75 (em uma escala de 0,0 a 1,0$)$. Isto é, ao observar casos sob a mesma condição, no mínimo três quartos dos casos deverão ser outliers para que ela seja considerada suficiente.

Para análise de necessidade, o valor de consistência deve ser mais rígido e será considerado o valor 1,0 . Isso significa dizer que, para uma condição ser considerada necessária, todos os casos outliers devem possuir essa condição. Pondera-se 
Quadro 1. Condições (variáveis explicativas) consideradas na análise fuzzy-set/QCA

\begin{tabular}{|c|c|c|c|c|}
\hline & & & & \\
\hline (fator & $\begin{array}{l}\text { ortalidade infantil } \\
\text { de } 1 \text { ano) por } \\
\text { idos vivos no ano } \\
\text { onte: PROADESS/ }\end{array}$ & taxa & $\begin{array}{l}\text { infantil } \\
\text { eceber } \\
s^{1}\end{array}$ & \multirow{10}{*}{ 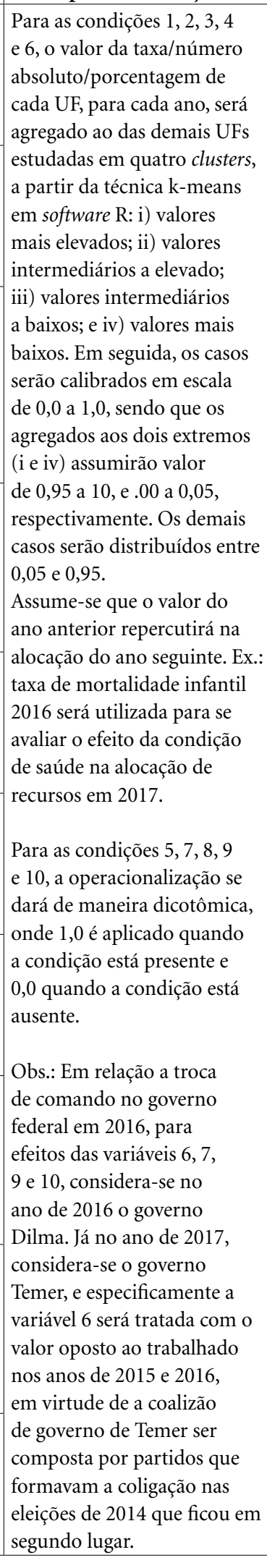 } \\
\hline $\begin{array}{l}\text { (fator } \\
\text { técnice }\end{array}$ & $\begin{array}{l}\text { nda média domiciliar per } \\
\text { pita no ano anterior. Fonte: } \\
\text { OADESS/Fiocruz. }\end{array}$ & $\begin{array}{l}\text { ECON (valor mais } \\
\text { elevado) }\end{array}$ & & \\
\hline $\begin{array}{l}\text { Capacidade } \\
\text { de oferta } \\
\text { (fator } \\
\text { técnico) }\end{array}$ & $\begin{array}{l}\text { úde } \\
\text { asto } \\
\text { e } \\
\text { Fonte: } \\
\text { partir } \\
z\end{array}$ & $\frac{\text { ofert }}{\text { baix }}$ & $\begin{array}{l}\text { dade } \\
\text { receita } \\
\text { icaria }\end{array}$ & \\
\hline $\begin{array}{l}\text { 4. População } \\
\text { SUS- } \\
\text { dependente } \\
\text { (fator }\end{array}$ & $\begin{array}{l}\text { ão } \\
\text { os } \\
\text { à } \\
\text { te: } \\
\text { ir }\end{array}$ & $\begin{array}{l}\text { pops } \\
\text { baix }\end{array}$ & & \\
\hline $\begin{array}{l}\text { de MA } \\
\text { capita } \\
\text { (fator }\end{array}$ & te: & $\underline{\text { histc }}$ & & \\
\hline (fa & 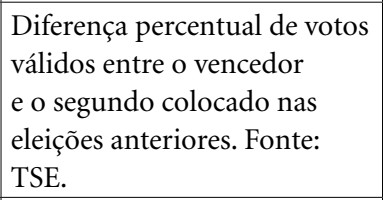 & vot & do & \\
\hline or & ser & COI & $\begin{array}{l}\text { deral } \\
\text { er }\end{array}$ & \\
\hline $\begin{array}{l}\text { istro } \\
\text { de de } \\
\text { da }\end{array}$ & $\begin{array}{l}\text { co da UF } \\
\text { Ministério }\end{array}$ & . & $\begin{array}{l}\text { F } \\
\text { ber }\end{array}$ & \\
\hline $\begin{array}{l}\text { do } \\
\text { alin }\end{array}$ & al. & $\begin{array}{l}\underline{\text { SEN }} \text { (condiçao } \\
\text { presente) }\end{array}$ & $\begin{array}{l}\text { nhada } \\
\text { ral } \\
\text { ceber } \\
2\end{array}$ & \\
\hline olítico) & $\begin{array}{l}\text { Preteito da capital da UF ser } \\
\text { alinhado com coalizão do } \\
\text { governo federal. Fonte: TSE } \\
\text { e publicação que sistematiza } \\
\text { coalizões }^{28} \text {. }\end{array}$ & presente) & 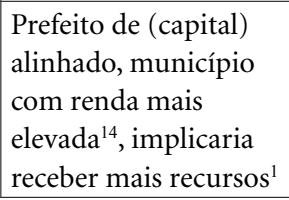 & \\
\hline
\end{tabular}

Fonte: Elaborado pelo autor. 
que a análise de necessidade é um pouco mais complexa e exige também observar a noção de relevância enquanto condição necessária. Não há um parâmetro bem definido sobre valor mínimo de relevância, pois isso depende do objeto em análise. Como este trabalho opta por fazer uma análise centrada nos casos outliers (são os casos mais extremos possíveis), é de se esperar valores relativamente baixos de relevância, sem que isso prejudique a discussão proposta. Se o valor de relevância da necessidade assumir valor maior que 0,5 , a condição será de fato considerada necessária. Se assumir valor abaixo de 0,5 , será feita ponderação, exigindo-se cautela na leitura do resultado, mas sem que isso elimine automaticamente a possiblidade de ser condição necessária. Por fim, como pode haver mais de uma condição ou uma combinação de condições associando resultados de suficiência e necessidade (preceito de multiplicidade causal), se for necessário fazer seleções, será escolhida a configuração com maior valor de cobertura, e que esteja condizente com o esperado a partir do referencial teórico e, consequentemente, disposto no Quadro 1.

Dito isso, serão rodados três modelos. Um para todos os casos em análise. Outro moderando os casos pela região geográfica, e por último um terceiro modelo moderando o fator tempo, até por conta de se esperar diferentes contextos políticos ao longo dos anos de análise.

\section{Resultados da análise quantitativa}

A Tabela 1 apresenta os valores de MAC per capita corrigidos para o ano de 2017, considerando uma série histórica de 15 anos, já classificando os casos a partir da técnica k-means de clusterização ano a ano, conforme per capita mais elevado (células pretas), per capita mais baixo (células cinzas) e per capita intermediário (células brancas)

Inicialmente, já se pode identificar que existem UFs que ao longo de toda a série histórica aparecem no grupo de per capita mais elevado: RS e MS. Por outro lado, AM é o único estado que sempre figurou no grupo de per capita mais baixo. Comparativamente, se considerarmos os últimos dez anos da série histórica (2008 a 2017), pode-se somar no grupo do per capita mais elevado PE e, no grupo do per capita mais baixo, PA e MA. Se consideramos apenas os últimos cinco anos (2013 a 2017), AC, TO e PR também se somam ao grupo do per capita mais elevado.

Outro destaque é que RN sai diretamente do grupo do per capita mais baixo para o per capita mais alto em 2017. O mesmo aconteceu com DF em 2009 e TO em 2005. Por fim, no ano de 2003, o menor per capita era do AM, no valor de R\$ 2,61, enquanto o maior era RJ, com R\$156,77. Já em 2004, o menor per capita de MAC era ES, com $\mathrm{R} \$ 27,74$, e o maior era SP, com R\$168,13. Em 2005, o menor per capita era RR, com R\$90,44, e o maior era RS, com R\$165,61. E, para comparar, em 2017, o menor per capita é AM, com R\$ 140,98 , e o maior é RN, com R\$271,84. Por fim, registra-se que o maior per capita de MAC registrado foi em 2014 em PE, no valor de R\$321,93.

Considerando as informações da Tabela 1 é inegável que houve uma profunda redistribuição desses recursos, especialmente envolvendo estados do Norte e Nordeste. A diferença entre os valores mais baixos e mais altos diminuíram consideravelmente ainda nos primeiros anos de análise, entre 2003 e 2005. Selecionando-se alguns dados emblemáticos, em 2014 e 2017 estados da região Nordeste apresentam os maiores valores de per capita. Ao longo dos diversos anos, TO em 2005 e RN em 2017 saíram diretamente do grupo de menor per capita para o de maior per capita. Dessa forma, pelos menos no que se refere à alocação de recursos federais, houve uma considerável diminuição das desigualdades regionais.

Observando-se agora o Gráfico 1, do tipo boxplot, nota-se a variação percentual do per capita de MAC a partir de 2004 em relação ao ano anterior, quando podemos identificar a existência de outliers (bolinhas brancas) em todos os anos, com exceção de 2011, que na verdade registrou um outlier de redução de recursos.

Em síntese, é evidente a discrepância da variação per capita em 2004 (em relação ao ano de 2003), pois existem três outliers cujas variações destoam de todos os demais outliers dos anos observados. Para facilitar a identificação dos casos outliers, a Tabela 2 sistematiza os valores de variação em termos percentuais, destacando os outliers que tiveram variações positiva para recebimento de recursos (células sombreadas de preto) e negativa (redução de recursos, com células sombreadas de cinza).

Complementando a análise boxplot com a Tabela 2, observa-se 22 casos outliers de variação positiva (aumento no recebimento de recursos fora do padrão) de 2004 a 2017. Desses 22, somente dois não envolvem UFs das regiões Norte e Nordeste. Foram ES, em 2005, que conforme dados da Tabela 1 teve o menor per capita de 2004, e DF, em 2009. Dessa forma, quando observamos especificamente UFs do Norte e Nordeste, apenas PA, AL, BA e CE não tiveram ampliação de recur- 


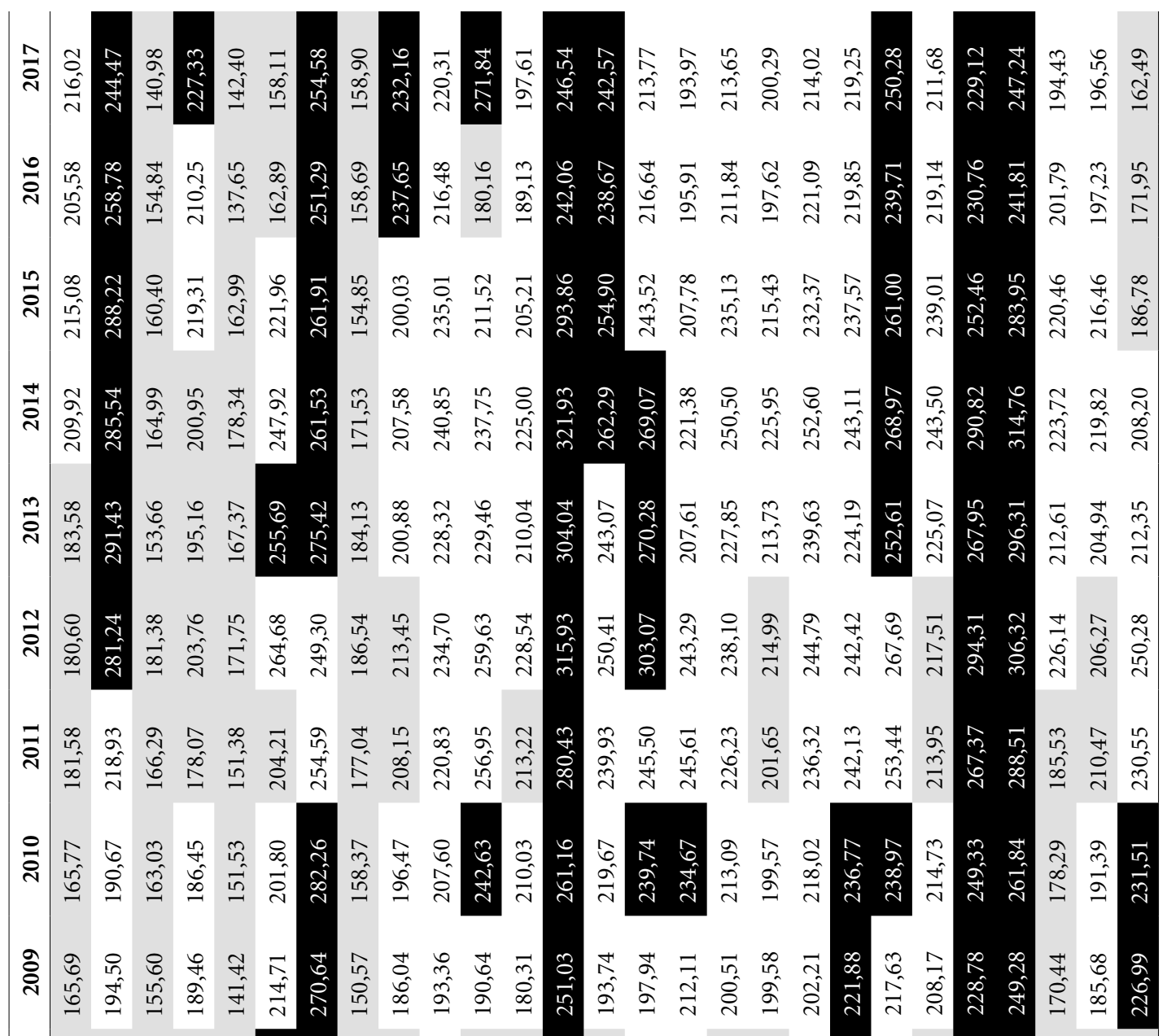

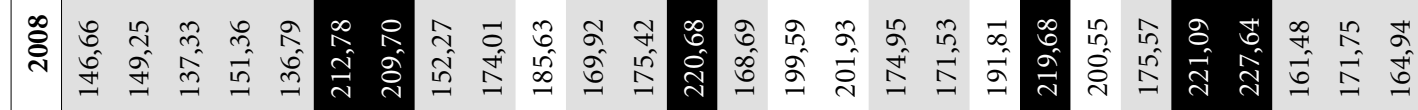

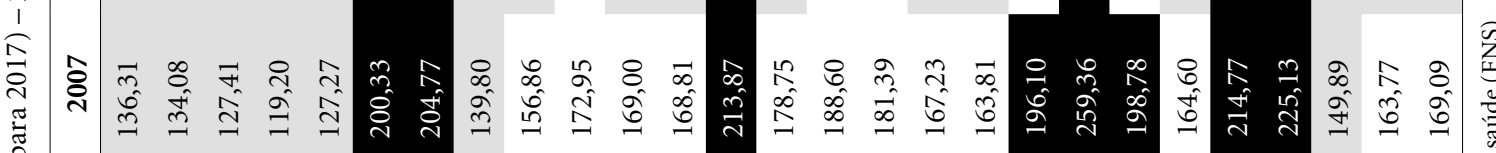

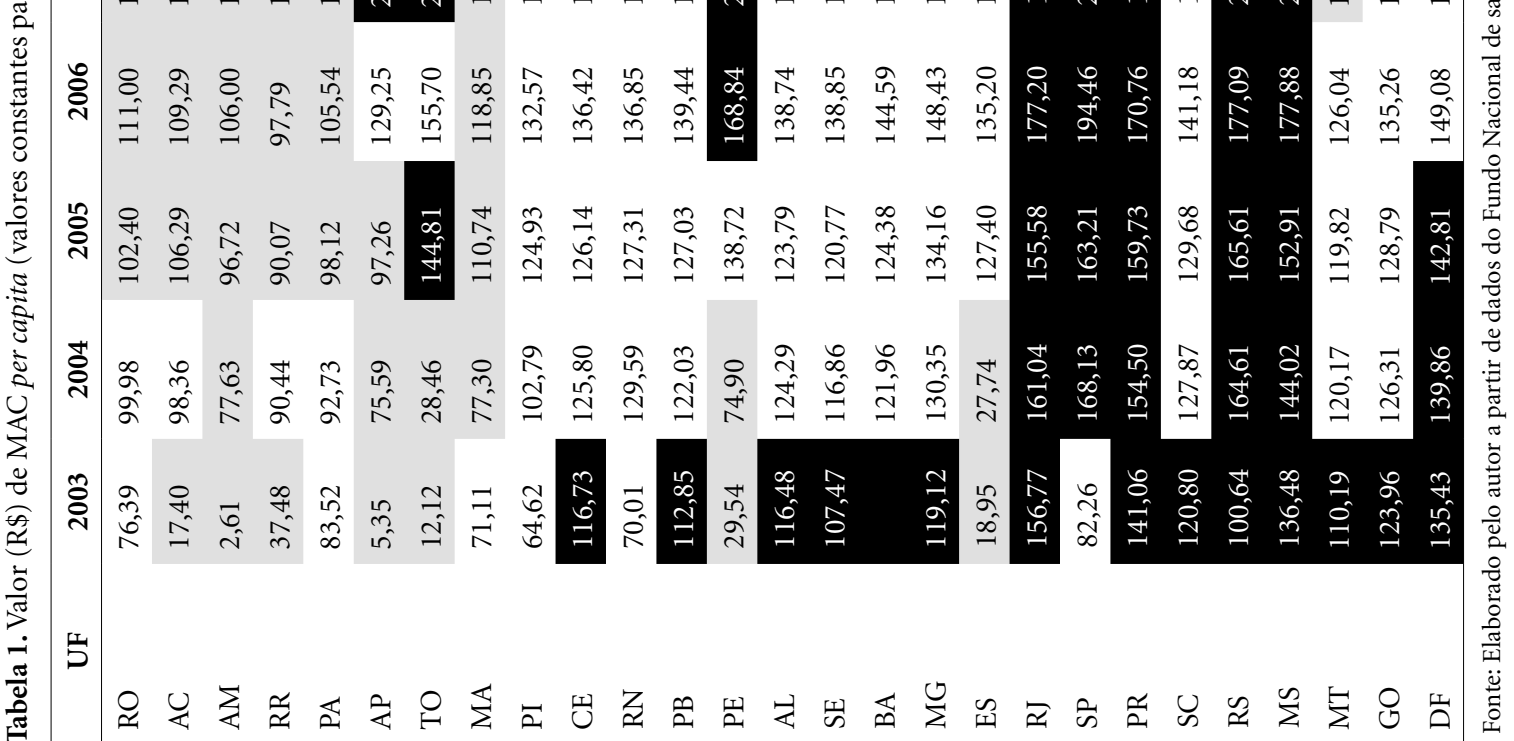




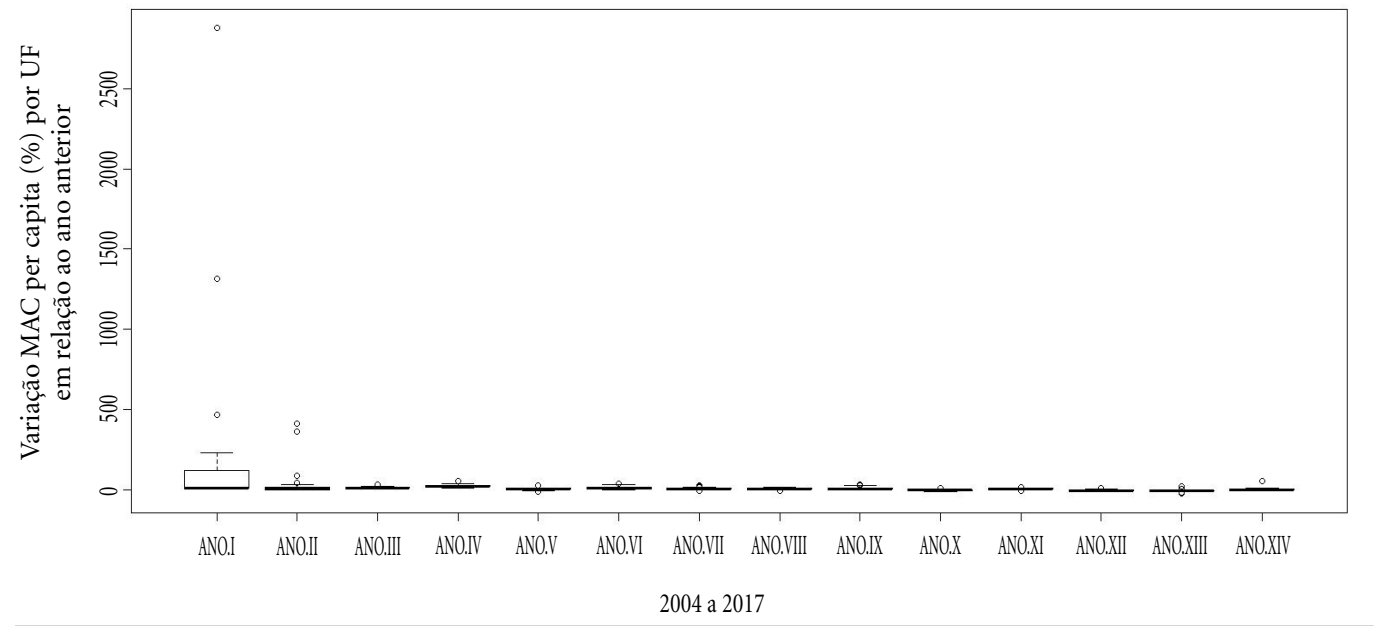

Gráfico 1. Variação MAC per capita (\%) por UF em relação ao ano anterior - 2004 a 2017.

Fonte: Elaborado pelo autor.

Tabela 2. Diferença MAC per capita (\%) em relação ao ano anterior (valores constantes).

\begin{tabular}{|c|c|c|c|c|c|c|c|c|c|c|c|c|c|c|}
\hline JF & 04 & 05 & 006 & 2007 & 2008 & 2009 & 010 & 011 & 2012 & 013 & 2014 & 2015 & 016 & 2017 \\
\hline $\mathrm{O}$ & & 43 & 39 & 2,80 & 59 & 298 & 005 & & $-0,54$ & 165 & 14,35 & 2,45 & & - \\
\hline C & 465,26 & & 82 & & & & & & 28,46 & & $-2,02$ & & & \\
\hline $11 \sqrt{1}$ & 9 & 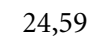 & 9,59 & & 7,78 & & 4,77 & & 9,08 & & 37 & $-2,78$ & & \\
\hline & o & & & & 26,97 & & 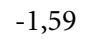 & & & & 2,97 & 9,13 & & \\
\hline & & & 56 & &, 48 & & & & & & & $-8,61$ & & \\
\hline 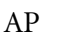 & 1313,31 & 28,66 & 32,89 & 55,00 & 6,21 & ,91 & $-6,01$ & 1,20 & 29,61 & $-3,40$ & $-3,04$ & 47 & & $-2,94$ \\
\hline TO & 134 & 100 & 7,52 & & 2,41 & & & & $-2,08$ & 10,48 & $-5,04$ & & & 31 \\
\hline MA & & 4 & 2 & & 2 & & & & 6 & & $-6,84$ & & 8 & \\
\hline IDI & 5 & 21,54 & 6 & & 10,93 & & 5,60 & & 2,55 & 9 & 3,34 & $-3,64$ & 18,81 & $-2,31$ \\
\hline $\mathrm{CE}$ & & & 5 & & 7,33 & & ונכ, & & 6,28 & & 49 & 2 & & 1,77 \\
\hline & & & 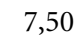 & & 0 & & 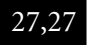 & & 1,04 & & 3,62 & 3 & & 50,89 \\
\hline & & & & & & & 10,10 & & & & (2, & & & \\
\hline & & & & & & & & & & & & & & \\
\hline$T$ & & & & & & & & & & & 91 & & & \\
\hline 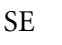 & & & & & 2 & $-0,82$ & 21,12 & & 23 & & $-0,45$ & $-9,49$ & & -1 , \\
\hline BA & & & 25 & & 11,32 & & 10,64 & & $-0,94$ & & 6,63 & & & $-0,99$ \\
\hline MC & & 2,93 &, 64 & 12 & 4,62 & 14 & & & 5,25 & & 9,94 & $-6,14$ & & 0,85 \\
\hline ES & 46,42 & 59,22 & 6,12 & 21,16 & 4,71 & 16,3 & 0,0 & 1. & 6,62 & -0 & 5,72 & $-4,66$ & $-8,27$ & 35 \\
\hline RJ & & & & & & & & & & & 41 & & & \\
\hline SP & & & 19,15 & & $-15,30$ & & & & & & 8,44 & & & $-0,27$ \\
\hline & & & & & & & & & & & & & & \\
\hline 8 & & & & & & & & & & & & & & $-3,40$ \\
\hline RS & . & 0,61 & 3 & 21,28 & 2,94 & 3 & 8, & 7,23 & 10,08 & -8 & 8,54 & $-13,19$ & & $-0,71$ \\
\hline MS & & & & & & & & & & & 23 & $-9,79$ & & 2,24 \\
\hline $\mathrm{M}^{\top}$ & & & & & & & & & 21,89 & & 5,22 & $-1,45$ & & $-3,65$ \\
\hline GO & & & & & 4,88 & & 3,08 & & $-2,00$ & & 7,26 & $-1,53$ & $-8,88$ & $-0,34$ \\
\hline $\mathrm{DF}$ & 3,27 & 2,11 & 39 & 13,43 & 2,46 & 7,62 & 1,99 & $-0,42$ & 8,56 & $-15,16$ & $-1,96$ & $-10,29$ & $-7,94$ & $-5,50$ \\
\hline
\end{tabular}

Fonte: Elaborado pelo autor a partir de dados do Fundo Nacional de saúde (FNS). 
sos a ponto de serem enquadrados como outliers em toda a série histórica. Por outro lado, se excluirmos da série histórica o ano de 2004, que até mesmo no Gráfico 1 destoa de todos os demais anos, $\mathrm{AM}$ se soma às demais quatro UFs e não figura como outlier de 2005 a 2017.

Dessa forma, evidencia-se que a alocação atípica de recursos (outliers) envolve especialmente estados das regiões Norte e Nordeste, chamando a atenção o fato de que, entre 2005 e 2017, das 16 UFs dessas regiões, apenas cinco não aparecem como outlier, enquanto AP, RN, RR e TO aparecem mais de uma vez como outliers.

Enfim, para compreender melhor o que explica esse fenômeno de alocação atípica de recursos do SUS, especificamente de MAC, a próxima seção objetivará dar respostas observando a associação de casos outliers com condicionantes técnicas e políticas. Dada a representatividade das regiões Norte e Nordeste nesse fenômeno, esse estudo optará por fazer a análise fuzzy-set/QCA centrada nas 16 UFs dessas regiões entre 2005 e 2017, excluindo-se da próxima análise os estados do Sul, Sudeste e Centro-Oeste, e também os dados de 2004, dada a inegável discrepância desse ano, que se relaciona a um nível de per capita muito abaixo de todas as demais UFs em 2003, como é o caso de $\mathrm{AM}(\mathrm{R} \$ 2,61)$. Isso posto, no ano de 2004, observando-se os casos outliers, nota-se um considerável foco na redução das desigualdades regionais, destacando-se um fator técnico: a defasagem em relação às demais UFs do país.

\section{Resultados da análise qualitativa}

A Figura 1 sistematiza os achados das oito análises realizadas: toda a amostra (M1); região Nordeste (M2.1); região Norte (M2.2); primeiro mandato do presidente Lula (M3.1); segundo mandato de Lula (M3.2); primeiro mandato da presidente Dilma (M3.3); segundo mandato de Dilma (M3.4); e o ano de 2017, com o presidente Temer (M3.5).

Ao todo, sete outliers dos 17 puderam ser explicados pela análise de condições suficientes. No que se refere às análises de necessidade, em todos os modelos foram identificadas condições necessárias, tendo duas com relevância de necessidade maior que 0,7 .

Observando-se o Modelo 1 (M1), identificamse como condições necessárias HISTOR, MINIST ou PREF, isto é, uma UF fazer parte de grupo de UFs com menor MAC per capita no ano anterior, ministro da Saúde no ano anterior ser político da
UF analisada ou prefeito da capital da UF ser alinhado com a coalizão do governo federal. Pondera-se que o valor de relevância dessa configuração é de 0,272 , exigindo um pouco mais de cautela para considerar de fato esse conjunto de condições como necessário para uma UF ser outlier. De toda forma, como apontado na metodologia, sendo o foco da análise os casos extremos mais emblemáticos, considera-se razoável que os valores de relevância sejam baixos.

Em complemento, nesse primeiro modelo foi possível identificar uma condição suficiente, isto é, basta ela está presente para que uma UF se torne outlier no recebimento de novos recursos de MAC. A condição em questão é MINIST (ministro da Saúde ser político da UF analisada no ano anterior). Dois casos estão relacionados a essa condição: PE em 2005 e PI em 2016. Esse achado por si só já demonstra ser possível que um ministro da Saúde direcione novos recursos para seu reduto político, apontando que as transferências do SUS estão sujeitas a explicações políticas, e não só técnicas - resultado alinhado a discussões recentes da ciência política ${ }^{20}$.

No M1 não se identificam condições suficientes para os demais 15 casos outliers, reforçando a necessidade das análises seguintes que moderam os dados pelas questões geográficas e temporais. No M2.1, região Nordeste, identificam-se como condições necessárias as mesmas do M1. Quanto às condições suficientes, além dos casos condicionados por MINIST, aparece como condição suficiente no caso do RN em 2017 a combinação HISTOR $^{\star}$ popsus ${ }^{\star}$ SEN, que representa uma interação entre fatores técnicos e políticos: histórico de MAC per capita e bancada do Senado alinhada, além de um valor baixo de população SUSdependente (o que pode estar mais relacionado indiretamente a um elevado nível socioeconômico). Observa-se que o sinal ${ }^{\star}$ significa multiplicação, portanto esse tipo de configuração deve ser lido considerando todas as três condições juntas (não são três condições alternativas, mas apenas uma para o resultado encontrado). Dessa forma, a leitura da combinação pode ser: em contextos de baixo valor de população SUS-dependente, o histórico defasado de MAC, juntamente com a bancada do Senado alinhada, é uma combinação suficiente para que uma UF seja outlier. No M2.2, região Norte, como condição necessária surge a configuração SEN ou PREF. E como condição suficiente, a combinação de fatores técnicos e políticos $\mathrm{HISTOR}^{\star} \operatorname{sen}^{\star} \mathrm{PREF}^{\star}$ vot, sugerindo a importância de prefeito de capital alinhado para o caso do TO em 2005. 


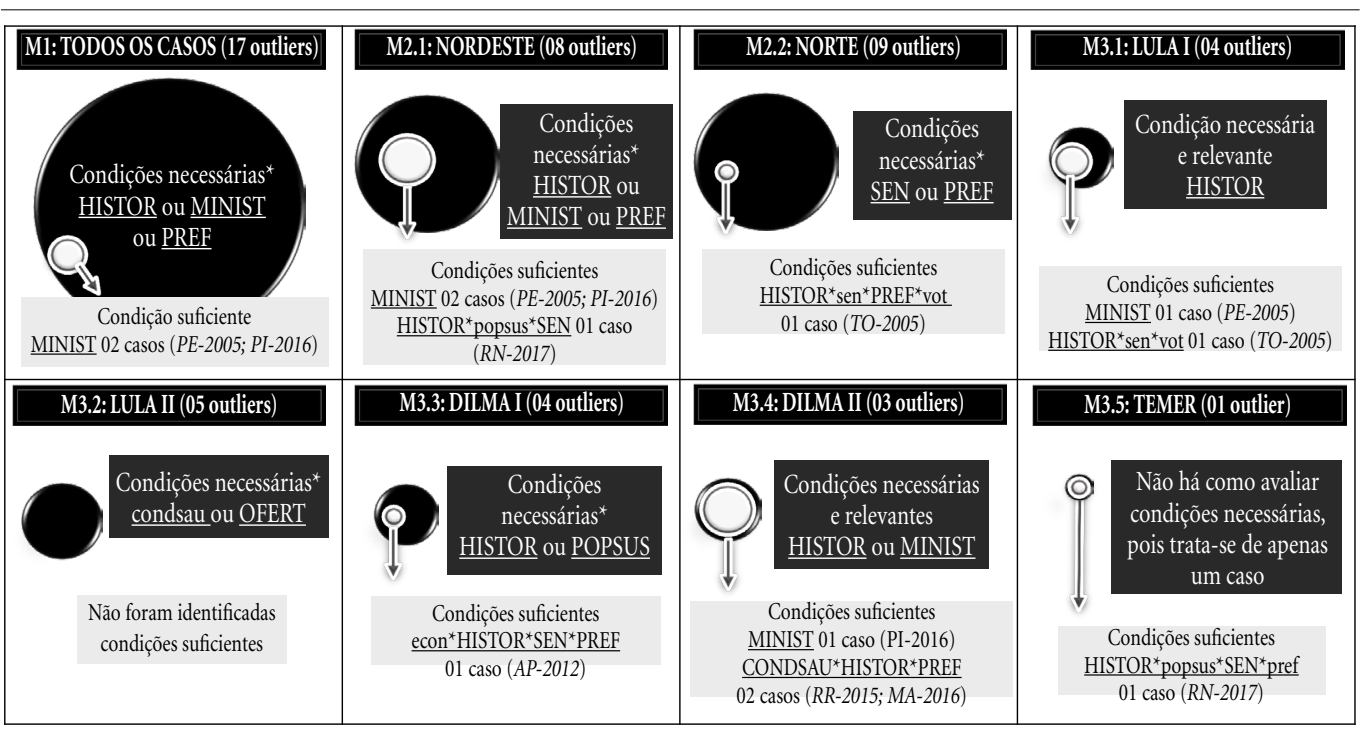

* condições necessárias, mas com valor de relevância relativamente baixo (ver discussão).

Figura 1. Resultados das análises de condições necessária e suficientes para os oito modelos testados, na forma de conjuntos.

Fonte: Elaborado pelo autor.

Ao avaliar os casos moderando-se pelo fator tempo (e mandato dos presidentes da República), cabe destaque que no M3.1, governo Lula 1, HISTOR figura como condição necessária com relevância considerável de 0,714. Nos modelos 3.2 e 3.3, Lula II e Dilma I, respectivamente, aparecem configurações necessárias com outros fatores técnicos, OFERT ou condsau e HISTOR ou POPSUS. Já no segundo mandato de Dilma (M3.4), surge a interação entre variáveis técnicas e políticas como condições necessárias e relevantes, HISTOR ou

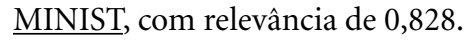

No que se refere a condições suficientes, moderando-se os achados pelo fator tempo, identificam-se explicações para sete dos 17 outliers. São os dois casos já explicados pela condição MINIST; o caso do TO em 2005, com fator técnico HISTOR $^{\star}$ sen $^{\star}$ vot; e outros quatro casos combinando condições técnicas e políticas: AP em 2012, econ ${ }^{\star} \mathrm{HISTOR}^{\star} \mathrm{SEN}^{\star} \mathrm{PREF} ; \mathrm{RR}$ em 2015 e MA em 2016 com a mesma combinação de condições suficientes no segundo governo da presidente Dilma, CONDSAU ${ }^{\star} \mathrm{HISTOR}^{\star} \mathrm{PREF}$; e o caso do RN em 2017, no governo Temer, com HISTOR $^{\star}$ popsus ${ }^{\star}$ SEN $^{\star}$ pref.

Em síntese, nos dois mandatos de Lula e no primeiro de Dilma, prevalecem fatores técnicos como condições necessárias para ser outlier no recebimento de recursos novos. E já no segundo mandato de Dilma, observa-se uma combinação de fatores técnicos e políticos como condições necessárias para ser outlier. No geral, destacamse como condições necessárias (isto é, são requisitos para que uma UF seja outlier) especialmente HISTOR e MINIST.

No que se refere às condições suficientes (isto é, a presença de determinada condição inevitavelmente leva uma UF a ser outlier), destaca-se MINIST como sendo a única condição que sozinha assegura o enquadramento de UFs como outlier. Outra condição relevante é HISTOR, que em conjunção com condições políticas, especialmente SEN e PREF, explica todos os outros outliers desse trabalho, sugerindo o enquadramento com o que se chama de "INUS condition"22,26, ou seja, uma condição que sozinha não garante que uma UF seja outlier mas, essa mesma condição, sendo parte necessária de uma combinação de condições suficientes. Isso demonstra a relevância da combinação de fatores técnicos e políticos para explicar a alocação atípica de recursos.

Dessa forma, os achados da presente análise demonstram que fatores políticos, e não só técnicos, são relevantes para explicar a alocação de recursos no SUS, em especial ministro da Saúde ser de origem da UF beneficiada e bancada do Sena- 
do e prefeito de capital alinhados. Isso indica que os condicionantes identificados nesse estudo, sobretudo os dois últimos, não são tão dissociados daqueles que explicam as TVUs ${ }^{2,5,6}$ e também reforçam os achados de estudos no campo da saúde em que fatores políticos explicam a distribuição de recursos para municípios ${ }^{1}$. No mais, o achado da relevância do cargo de ministro da Saúde está bem alinhado com as discussões mais recentes da ciência política sobre o uso de cargos estratégicos para direcionamento de recursos ${ }^{20}$.

\section{Conclusão}

Em suma, entende-se que o presente trabalho cumpre seu papel ao explorar a dimensão de fatores políticos como condicionantes dos repasses de recursos federais do SUS, demonstrando que os critérios de repasses de recursos da saúde não são tão dissociados das transferências voluntárias quanto poderia se imaginar.

Em que pese ter ficado claro na análise quantitativa que a alocação de recursos diminuiu consideravelmente as distorções de valores per capita dos recursos de MAC para estados das regiões Norte e Nordeste, reduzindo as desigualdades regionais, ficou notória na análise qualitativa a in- terveniência de fatores políticos condicionando a alocação de recursos. Destaca-se o resultado encontrado em que um político da região Norte ou Nordeste ser ministro da Saúde assegura o direcionamento de novos recursos, de forma atípica, para o seu reduto político - achado possível de ser observado, dado o foco de análise qualitativa em casos outliers. Ao mesmo tempo, é relevante a identificação da distribuição de recursos do SUS estar condicionada à combinação de fatores técnicos e políticos - outro aspecto observado em decorrência da análise qualitativa empreendida.

Por outro lado, o foco nos casos outliers permitiu tão somente uma análise exploratória acerca dos efeitos de condicionantes políticos na alocação de recursos do SUS, o que contribui na proposição de hipóteses e reflexões teóricas. Portanto, futuras pesquisas nessa temática são desejáveis, especialmente considerando outros objetos de análise, tais como observação de casos que não sejam extremos, outras regiões geográficas além de Norte e Nordeste e também análises sobre investimentos, políticas e programas específicos do Ministério da Saúde, podendo-se avaliar o efeito de eventuais fatores políticos nos resultados na saúde da população - aspecto, por exemplo, que seria bem relevante. 


\section{Referências}

1. Simão JB, Orellano VIF. Um estudo sobre a distribuição das transferências para o setor de saúde no Brasil. Estudos Econômicos 2015; 45(1):33-63.

2. Arretche M, Rodden J. Política distributiva na Federação: estratégias eleitorais, barganhas legislativas e coalizões de governo. Dados 2004; 47(3):549-576.

3. Ferreira IFS, Bugarin MS. Transferências voluntárias e ciclo político-orçamentário no federalismo fiscal brasileiro. Revista Brasileira de Economia 2007; 61(3):271-300

4. Piola SF. Transferências de recursos federais do Sistema Único de Saúde para estados, Distrito Federal e municípios: os desafios para a implementação dos critérios da Lei Complementar no 141/2012. Brasília: Ipea; 2017.

5. Soares MM, Melo BG. Condicionantes políticos e técnicos das transferências voluntárias da União aos municípios brasileiros. Revista de Administração Pública 2016; 50(4):539-562.

6. Amorim Neto O, Simonassi AG. Bases políticas das transferências intergovernamentais no Brasil (19852004). Brazilian Journal of Political Economy 2013; 33(4):704-725.

7. Jaccoud L, Vieira F. Federalismo, integralidade e autonomia no SUS: desvinculação da aplicação de recursos federais e os desafios da coordenação. Brasília: Ipea; 2018.

8. Lima LDD. Conexões entre o federalismo fiscal e o financiamento da política de saúde no Brasil. Cien Saude Colet 2007; 12(2):511-522.

9. Souza RRD. Redução das desigualdades regionais na alocação dos recursos federais para a saúde. Cien Saude Colet 2003; 8(2):449-460.

10. Junior AP, Mendes AN. O Fundo Nacional de Saúde e a prioridade da média e alta complexidade. Argumentum 2015; 7(2):161-177.

11. Ugá MA, Piola SF, Porto SM, Vianna SM. Descentralização e alocação de recursos no âmbito do Sistema Único de Saúde (SUS). Cien Saude Colet 2003; 8(2):417-437.

12. Barros MED, Piola SF. O financiamento dos serviços de saúde no Brasil. In: Marques RR, Piola SF, Roa AC, organizadores. Sistema de Saúde no Brasil: organização e financiamento. Rio de Janeiro: ABrES; Brasília: Ministério da Saúde; OPAS/OMS no Brasil. 2016. p. 101-138.

13. Vazquez DA. Mecanismos institucionais de regulação federal e seus resultados nas políticas de educação e saúde. Dados 2014; 57(4):969-1005.

14. Arretche M. Financiamento federal e gestão local de políticas sociais: o difícil equilíbrio entre regulação, responsabilidade e autonomia. Cien Saude Colet 2003; 8(2):331-345.

15. Marques RM, Mendes A. O financiamento do Sistema Único de Saúde e as diretrizes do Banco Mundial. In: Pereira JMM, Pronko M. A demolição de direitos: um exame das politicas do Banco Mundial para a educação e a saúde (1980-2013). Rio de Janeiro: Escola Politécnica de Saúde Joaquim Venâncio; 2014. p. 275-293.

16. Guerra M. Modelo de alocação de recursos do sistema único de saúde para organizações hospitalares: serviços de alta complexidade [tese]. Universidade de Brasília, Brasília; 2013.
17. de Paiva AB, Gonzalez RHS, Leandro JG. Coordenação federativa e financiamento da política de saúde: mecanismos vigentes, mudanças sinalizadas e perspectivas para o futuro. Novos estud. CEBRAP 2017; 36(2):55-81.

18. Brasil. Portaria de consolidação No 6 , de 28 de setembro de 2017 [e alterações]. Consolidação das normas sobre o financiamento e a transferência dos recursos federais para as ações e os serviços de saúde do Sistema Único de Saúde; 2017. [acessado 2019 Abr 30]. Disponível em: http://bvsms.saude.gov.br/bvs/saudelegis/gm/2017/prc0006_03_10_2017.html

19. Banco Mundial. Governança no Sistema Único de Saúde (SUS) do Brasil. Melhorando a qualidade do gasto público e gestão de recursos. Washington (DC): Banco Mundial; 2007.

20. Lopez FG, organizador. Cargos de confiança no presidencialismo de coalizão brasileiro. Brasília: Ipea; 2015.

21. Creswell JW. Research design: Qualitative, quantitative, and mixed methods approaches. Thousand Oaks: Sage publications; 2014.

22. Goertz G, Mahoney J. A tale of two cultures: qualitative and quantitative research in the social sciences. Princeton: Princeton University Press; 2012.

23. King G, Keohane RO, Verba S. Designing social in quiry: Scientific inference in qualitative research. Princeton: Princeton university press; 1994.

24. R Core Team R: A language and environment for statistical computing. R Foundation for Statistical Computing, Vienna, Austria; 2019 Disponível em: https:// www.R-project.org/.

25. Ragin CC. Fuzzy-set social science. Chicago: Chicago University Press; 2000.

26. Ragin CC. Redesigning social inquiry: fuzzy sets and beyond. Chicago: University of Chicago Press; 2008.

27. Dusa A. QCA with R. A comprehensive resource. New York: Springer International Publishing; 2019.

28. Pereira C. Medindo a governabilidade no Brasil: o presidencialismo de coalizão nos governos FHC, Lula e Dilma. 87 f., il. [dissertação]. Universidade de Brasília, Brasília; 2017.

29. Schneider C, Wagemann C. Set-theoretic methods for the social sciences. Cambridge: Cambridge University Press; 2012.

30. Kahwati LC, Kane HL. Qualitative comparative analysis in mixed methods research and evaluation (Vol. 6). Thousand Oaks: SAGE Publications; 2018.

Artigo apresentado em 31/05/2019

Aprovado em 07/02/2020

Versão final apresentada em 09/02/2020

Editores-chefes: Romeu Gomes, Antônio Augusto Moura da Silva 
